\title{
Nonlinear Band Expansion and Nonnegative Matrix Underapproximation for Unsupervised Segmentation of a Liver from a Multi-phase CT image
}

\author{
Ivica Kopriva $^{1 *}$, Xinjian $\mathrm{Chen}^{2}$ and Jianhua $\mathrm{Yao}^{2}$ \\ ${ }^{1}$ Division of Laser and Atomic Research and Development, Ruđer Bošković Institute, Bijenička cesta \\ 54, P.O. Box 180, Zagreb, Croatia \\ ${ }^{2}$ Department of Radiology and Imaging Sciences, National Institutes of Health, Bethesda, Maryland 20892, \\ USA \\ e-mail: ikopriva@irb.hr, chenx6@mail.nih.gov,jyao@cc.nih.gov
}

\begin{abstract}
A methodology is proposed for contrast enhanced unsupervised segmentation of a liver from a twodimensional multi-phase CT image. The multi-phase CT image is represented by a linear mixture model, whereupon each single-phase CT image is modeled as a linear mixture of spatial distributions of the organs present in the image. The methodology exploits concentration and spatial diversities between organs present in the image and consists of nonlinear dimensionality expansion followed by matrix factorization that relies on sparseness between spatial distributions of organs. Dimensionality expansion increases concentration diversity (contrast) between organs. The methodology is demonstrated on an experimental three-phase CT image of a liver of two patients.
\end{abstract}

Keywords: Multi-phase CT image, liver delineation, unsupervised segmentation, nonnegative matrix factorization.

\section{INTRODUCTION}

The purpose of this paper is development of a method for contrast-enhanced unsupervised segmentation of the multi-phase CT image of a liver. Good contrast between the liver and organs surrounding it is of crucial importance for accurate delineation of the liver boundaries. However, due to the low contrast of the soft tissue computerized liver segmentation is still a challenging problem. For contrast enhancement low-dose non-contrast CT image is accompanied by contrast enhanced CT scans (arterial and portal-venous scans in this paper). It is however possible to further enhance contrast between organs by means of image segmentation methods, [1,2]. Here, we propose novel method for unsupervised segmentation of the multiphase CT image. The method relies on representation of the multi-phase CT image by means of a linear mixture model (LMM), whereas the multi-phase CT image is modeled as a product of matrix of concentration (or density) profiles and matrix of spatial distributions of the organs present in the image (the liver and the organs surrounding it). Unsupervised segmentation problem is thus converted into matrix factorization problem. Accuracy and uniqueness of matrix factorization depends on the fulfillment of constraints imposed on spatial distributions of organs. For an example, independent component analysis (ICA)-based approach to LMM-based segmentation of CT image, [1], presumed that spatial distributions of the organs present in the CT image are mutually statistically independent. However, contrast between organs also depends on the level of similarity of the concentration profiles of the organs across the phase images. In principle, the smaller number of contrast enhanced phase images implies the greater level of similarity between concentration profiles of the organs. When this occurs statistical independence between spatial distributions of the organs also decreases. This phenomenon has been discussed extensively in the context of

*ikopriva@irb.hr, phone: +38514571286; fax: +38514680104. 
the LMM-based unsupervised segmentation of the low-dimensional multi-spectral image, [3-6]. Therefore, the main objective of this paper is an experimental demonstration of nonlinear band expansion-based matrix factorization method for contrast enhanced unsupervised multi-phase CT image decomposition. Among several approaches to factorize the matrix of the band expanded CT image we have considered dependent component analysis (DCA) and sparseness-based matrix factorization methods. DCA compensates partially statistical dependence problem caused by similarity of the concentration profiles of the organs, while sparseness assumes no overlap between spatial distributions of the organs present in the CT image.

\section{MATERIALS AND METHODS}

For the segmentation purpose multi-phase CT image is represented in a form of the LMM

$$
\mathbf{X}=\mathbf{A S}
$$

where $\mathbf{X} \in R_{0^{+}}^{I_{3} \times I_{1} I_{2}}$ represents multi-phase $\mathrm{CT}$ image consisting of $I_{3}$ phase images and $I_{1} I_{2}$ pixels, $\mathbf{A} \in R_{0^{+}}^{I_{3} \times J}$ represents matrix of density profiles of the $J$ organs present in the image, that is also known as a mixing matrix, and $\mathbf{S} \in R_{0^{+}}^{J \times I_{1} I_{2}}$ represents matrix of spatial distributions of $J$ organs present in the image. Due to the nature of the problem all model variables in (1) are nonnegative. The LMM-based representation has been successfully used recently for unsupervised segmentation of multi-spectral image, [3-6], as well as for blind extraction of analytes from mixtures spectra in NMR spectroscopy [7,8], mass spectrometry [7,9] and FT-IR spectroscopy [10]. In multi-spectral imaging A represents matrix of spectral profiles of the objects present in the image, while $\mathbf{S}$ represents matrix of spatial distributions of the same objects. In application arising in chemometrics, $\mathbf{A}$ is interpreted as a matrix of concentration profiles of analytes present in the mixture, while $\mathbf{S}$ represents their spectral profiles. Thus, the LMM-based representation of the multi-phase CT image combines interpretations of the LMM in multi-spectral imaging and chemometrics.

Matrix factorization problem implied by LMM (1) has infinitely many solutions unless additional constraints are imposed on model variables $\mathbf{A}$ and $\mathbf{S}$. This is due to the fact that representation (1) and representation $\mathbf{X}=\mathbf{A} \mathbf{T} \mathbf{T}^{-1} \mathbf{S}$ are equivalent from the data matrix $\mathbf{X}$ point of view, whereas $\mathbf{T}$ is any invertible matrix. Thus, infinitely many pairs $(\mathbf{A}, \mathbf{S})$ can give rise to $\mathbf{X}$. To achieve solutions which are meaningful, i.e. have physical interpretation, factorization indeterminacies must be reduced to $\mathbf{T T}^{-1}=\mathbf{P} \Lambda$, whereas $\mathbf{P}$ is permutation matrix and $\Lambda$ is diagonal matrix. These are standard indeterminacies in blind source separation and can be achieved if either sparseness, [5,7-9], or statistical independence, [1,3,6], constraints are imposed on $\mathbf{S}$. Sparseness constraints imply that at each pixel location $\left(i_{1}, i_{2}\right)$ only few organs exists. In CT imaging, where pixel footprint is small, it is justified to assume that only one organ occupies each pixel footprint.

Described interpretation of the LMM (1) implies that concentration similarity of the organs $\mathbf{s}_{m}$ and $\mathbf{s}_{n}$ affects the condition number of the mixing matrix A. This is because the corresponding column vectors $\mathbf{a}_{m}$ and $\mathbf{a}_{n}$ become close to collinear. An equivalent problem exists in multi-spectral imaging that is caused by spectral similarity between the objects [3]. Thus, in addition to deteriorating conditioning of the mixing matrix density similarity between the organs makes also their spatial distributions statistically dependent. That is why DCA, [3,6], is necessary when statistical independence is used as a constraint for factorization of the CT image matrix. The density similarity caused problem can be also alleviated by introducing additional phase-contrast images. This, however, is mostly not a matter of choice, but dictated by the CT imager available. It is, therefore, of great importance for the contrast enhancement purpose that prior to matrix factorization of the LMM (1) the nonlinear band expansion (NBE), [3,11,2], is used to improve conditioning of the matrix of concentration profiles. This actually produces contrast-enhancement effect. The idea of using NBE for image segmentation has been first used in [11,12] to increase number of spectral bands when number of objects (sources) present in the multi-spectral image exceeds number of spectral bands available. However, it has been realized in [3], that NBE also improves conditioning of the mixing 
matrix which becomes important when objects (organs in multi-phase CT image) have similar concentration profiles.

Let $\left\{\mathbf{X}_{i_{3}} \in R_{0+}^{I_{1} \times I_{2}}\right\}_{i_{3}=1}^{3}$ be the original three-phase CT image comprised of non-contrast, arterial and portal-venous phase images. Through NBE we introduce new sets of images: set of auto-correlated phase images $\left\{\mathbf{X}_{i_{3}}^{2}\right\}_{i_{3}=1}^{3}$, and set of cross-correlated phase images $\left\{\mathbf{X}_{i_{3}} \mathbf{X}_{j_{3}}\right\}_{i_{3}=1, j_{3}=i_{3}+1}^{2,3}$. Hence, the NBE multi-phase CT image is then obtained as:

$$
\overline{\mathbf{X}} \approx \overline{\mathbf{A}} \mathbf{S}
$$

where $\overline{\mathbf{X}}_{(3)} \in R_{0+}^{9 \times I_{1} I_{2}}, \overline{\mathbf{A}} \in R_{0+}^{9 \times J}$ and $\mathbf{S} \in R_{0+}^{J \times I_{1} I_{2}}$. Thus, original three-phase CT image is transformed into "nine-phase" image. As it has been shown in [3], the source matrix in (1) and (2) remains the same if sources $\left\{\mathbf{s}_{j}\right\}_{j=1}^{J}$ do not overlap (as discussed previously this assumption is justified for LMM-based representation of the CT image) and are quasi-binary: $s_{j} \in\{0,1\}$, (this assumption is justified for reasonably good segmentation).

In this paper we demonstrate contrast-enhanced unsupervised segmentation of the liver from the three-phase CT image by applying two methods to factorize the NBE three-phase CT image (2): DCA and nonnegative matrix underapproximation (NMU). The DCA approach transforms the NBE CT image (2) by means of statistical-independence-enhancement linear transform $T$ :

$$
T(\overline{\mathbf{X}}) \approx \overline{\mathbf{A}} T(\mathbf{S})
$$

such that $T\left(\mathbf{s}_{m}\right)$ and $T\left(\mathbf{s}_{n}\right)$ are more statistically independent than $\mathbf{s}_{m}$ and $\mathbf{s}_{n}$. The ICA algorithm is then applied on (3) to estimate $\overline{\mathbf{A}}$, while $\mathbf{S}$ is obtained from (2) by $\mathbf{S} \approx \overline{\mathbf{A}}^{\dagger} \overline{\mathbf{X}}$. Typical choices for $T$ include innovations, [13], wavelet packets, [14], or high-pass filtering, [6]. The innovations transform and statistically efficient version of the FastICA algorithm, [15], have been used in this paper to implement the DCA-based factorization. The NMU, [16], is introduced very recently as a refinement of the nonnegative matrix factorization (NMF) algorithms, towards sparse factorization of $\overline{\mathbf{X}}$ in (2). In addition to nonnegativity constrains imposed on $\overline{\mathbf{A}}$ and $\mathbf{S}$, as it is done by all NMF algorithms, [17], the cost function $D(\overline{\mathbf{X}} \| \overline{\mathbf{A}} \mathbf{S})=\|\overline{\mathbf{X}}-\overline{\mathbf{A}} \mathbf{S}\|_{2}^{2}$ is minimized imposing an underapproximation constraint on $\overline{\mathbf{A}}$ and $\mathbf{S}$ : $\overline{\mathbf{A}} \mathbf{S} \leq \overline{\mathbf{X}}$. Due to last constraint, NMU decomposition of (2) naturally generates sparser solution than NMF, without imposing direct sparseness constrains on the rows of $\mathbf{S}$. To enforce sparseness of the solution even further $\ell_{1}$-norm version of $D(\overline{\mathbf{X}} \| \overline{\mathbf{A}} \mathbf{S})$ can be considered as well. The NMU-based segmentation results shown in section 3 are obtained with $\ell_{2}$-norm version of $D(\overline{\mathbf{X}} \| \overline{\mathbf{A}} \mathbf{S})$.

\section{RESULTS}

We demonstrate proposed approach to liver segmentation on the three-phase CT images of the two patients. Figure 1 shows three-phase CT image of the liver and surrounding organs of the first patient where in the top are row: non-contrast, arterial and portal-venous phase images, and associated isolines of the estimated external energy of the vector field convolution (VFC), $[18,19]$, are shown in the bottom row. As seen from isolines the liver cannot be localized. Figure 2 shows segmentation result obtained by applying the NMU algorithm to the NBE version of the three-phase CT image shown in Figure 1, while corresponding results obtained by DCA algorithm are shown in Figure 3. The sparseness-based NMU yielded anatomically and 
clinically more meaningful components than DCA, although both of them yielded liver related component shown correspondingly in the cluster b) in Figure 2 and middle of the bottom row in Figure 3. Yet, the NMU-segmented image has better contrast, that is further confirmed in Figure 4 where isolines for the NMU- and DCA- segmented images of the liver are shown. From the NBU-segmented image the liver area is localized better than in the case of DCA-segmented image as well as than in the case of the original threephase CT image shown in Figure 1. Here we would like to comment on the ICA-based segmentation of the liver from the four-phase CT image in [1]. Adding one contrast-enhancement phase more increases significantly level of dissimilarity between density profiles of the liver and non-liver organs in the image. Thus, all the problems associated with it are much less present when the four-phase CT image is used, as opposed to the three-phase CT image that is used herein. Figure 5 shows three-phase CT image of the liver and surrounding organs of the second patient in the top row, as well as associated isolines of the estimated external energy of the VFC in the bottom row. Note, that portal-venous phase image exhibited better liverrelated contrast than in the case of the first patient. This is visible on the associated isolines image. Segmentation results obtained by the NBE-NMU algorithm are shown in Figure 6. DCA-based results are not shown due to the reason of inferiority already demonstrated in the case of the first patient. By analogy with Figure 4, we show in Figure 7 an image of isolines of the NBE-NMU segmented image shown in Figure 6 , left in the cluster b). The isolines yield similar result as the one obtained on original portal-venous phase CT image shown in Figure 5. Note, however, that ribs and vessels are better suppressed on segmented image shown in Figure 6 (left in the cluster b) than original portal-venous image (rightmost in Figure 5 in a first row).
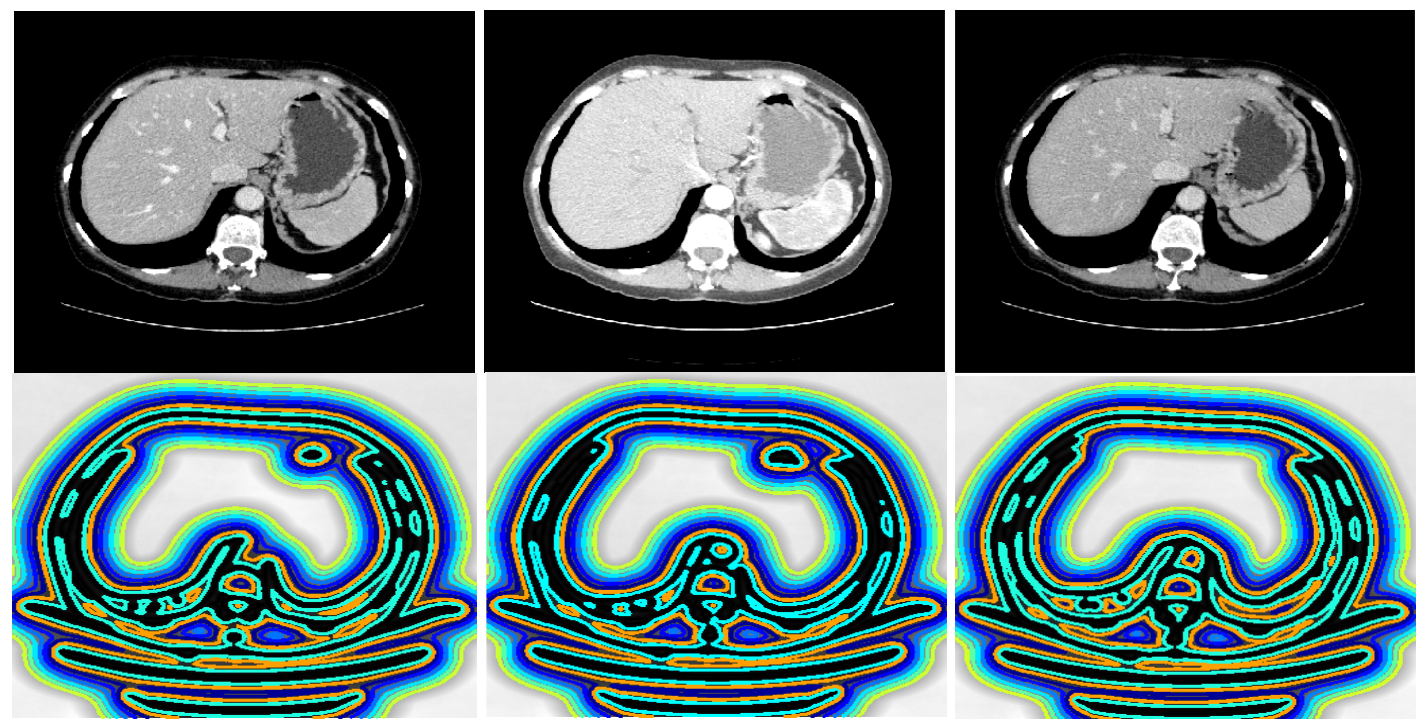

Figure 1. Top row: the three-phase CT image of the liver of the first patient. From left to right: non-contrast, arterial phase and portal-venous phase. Bottom row: isolines of the estimated external energy of the vector field convolution (VFC) for the three-phase CT images. 
a)

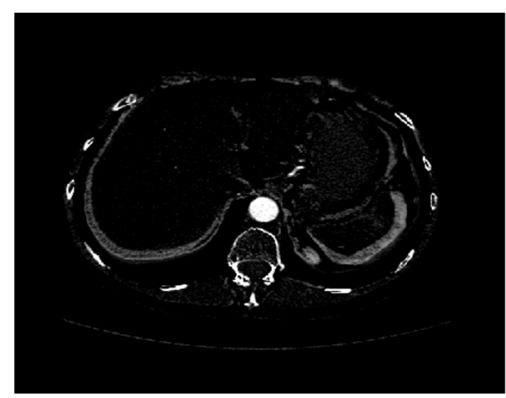

d)

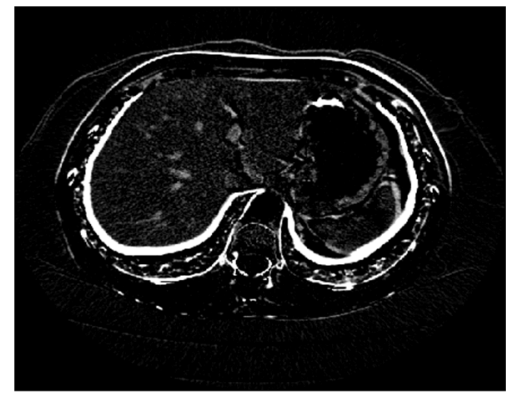

b)
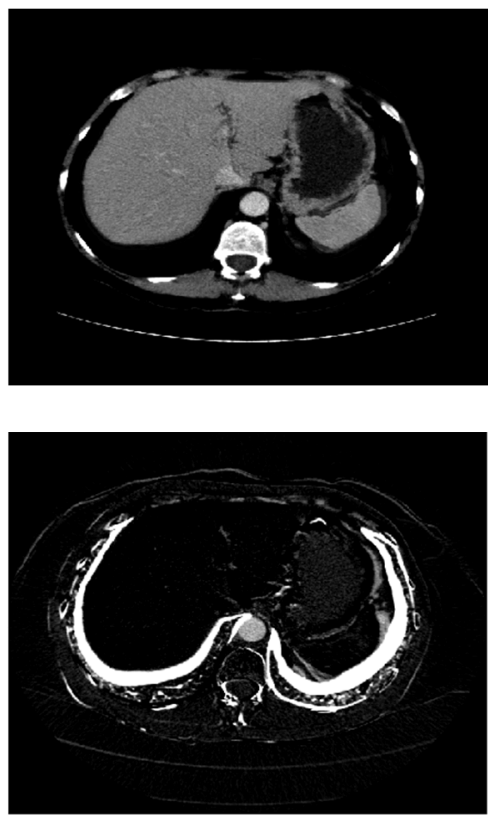

c)
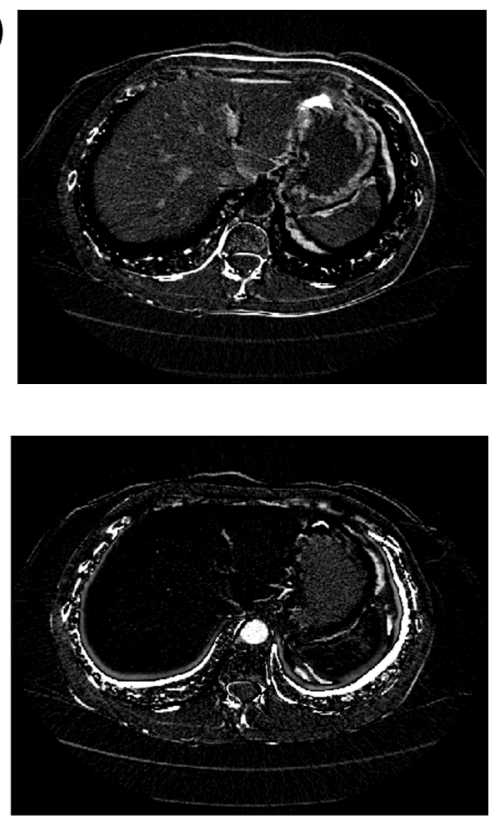

Figure 2. $\ell_{2}$-norm based NMU segmentation of the NBE three-phase CT image shown in Figure 1. Clinically and anatomically meaningful components were obtained and organized in four clusters where: a) vessels and rib are included; b) liver, spleen, spine and rib are included; c) spine and rib are included; d) lung is included.
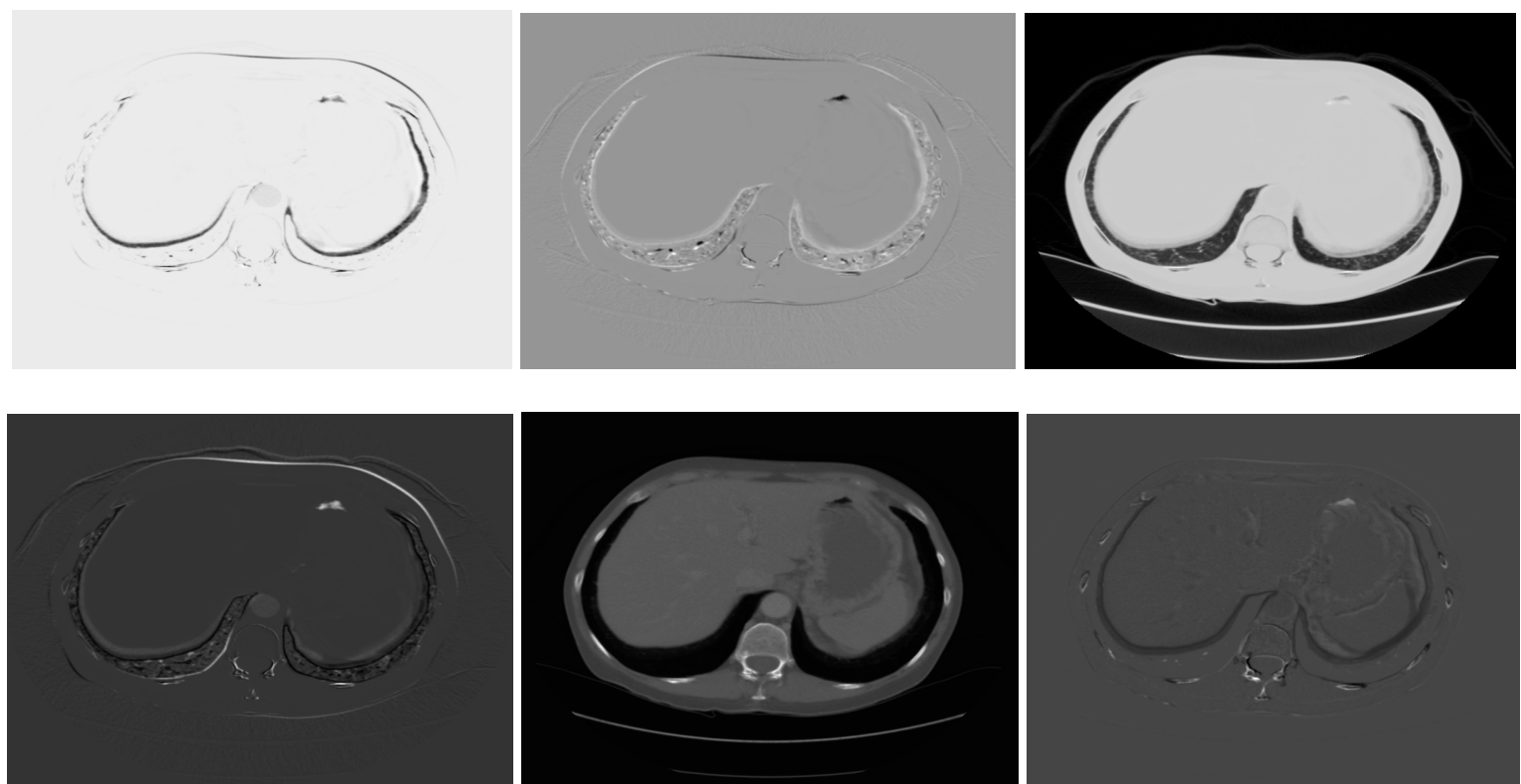

Figure 3. DCA-based segmentation of the NBE three-phase CT image shown in Figure 1. Clinical and anatomical interpretation of the components seems to be less meaningful than that of the NMU-obtained components shown in Figure 2. The liver related component is in the middle in the bottom row. 


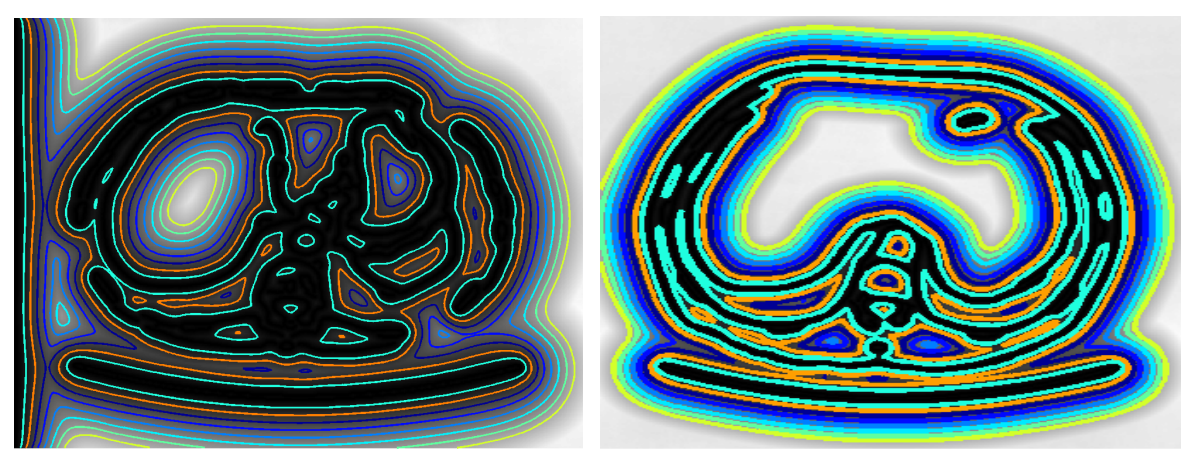

Figure 4. Isolines of the liver related segmented images by NMU (left) and DCA (right).
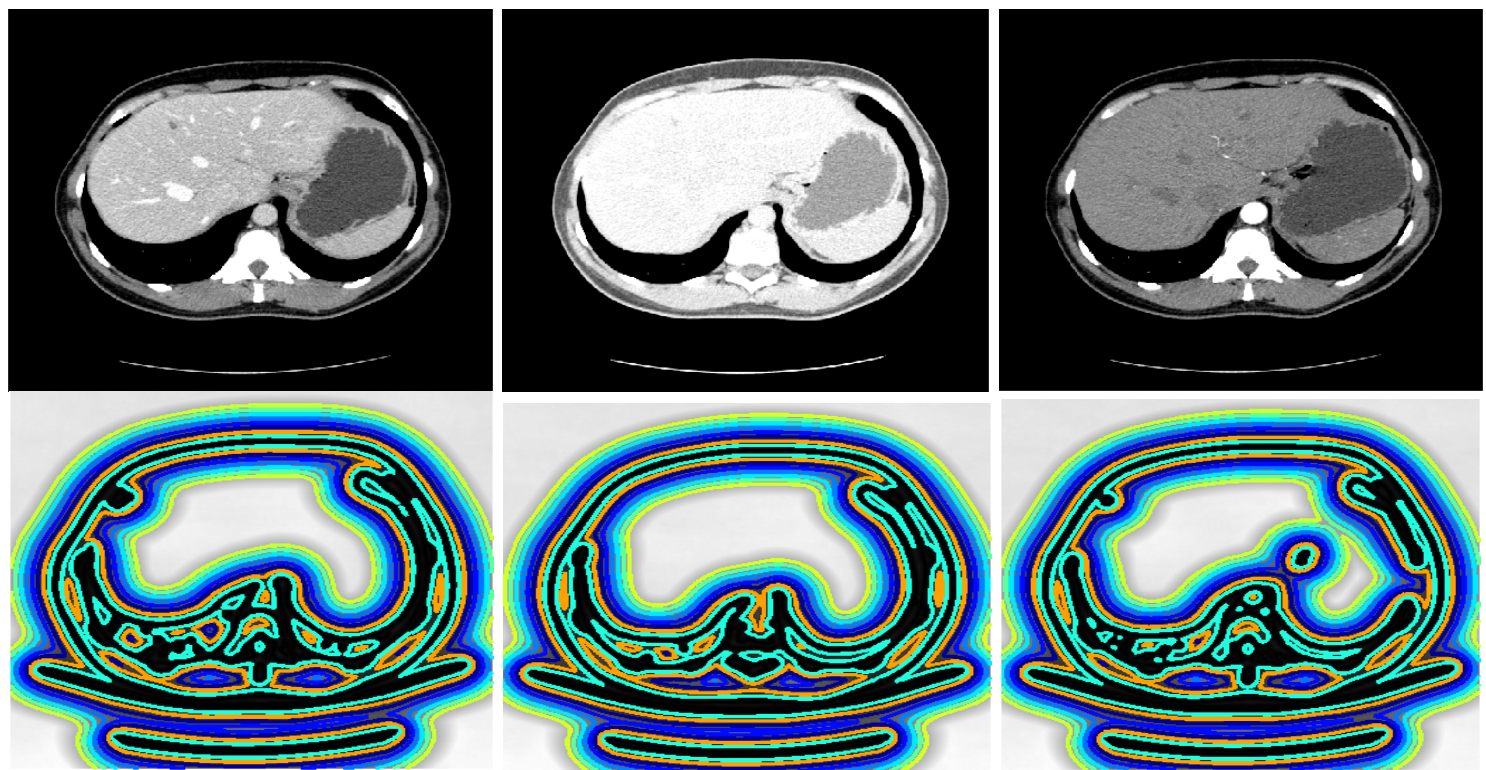

Figure 5. Top row: the three-phase CT image of the liver of the second patient. From left to right: noncontrast, arterial phase and portal-venous phase. Bottom row: Isolines of the estimated external energy of the vector field convolution (VFC) for the three-phase CT images. 
a)

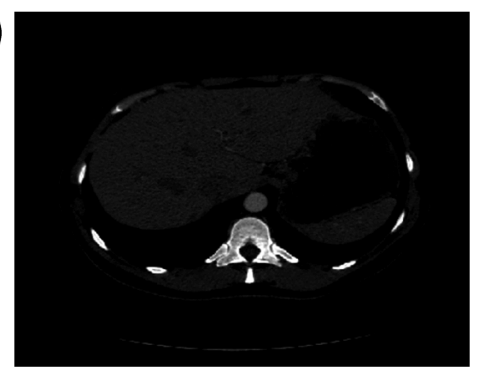

c)

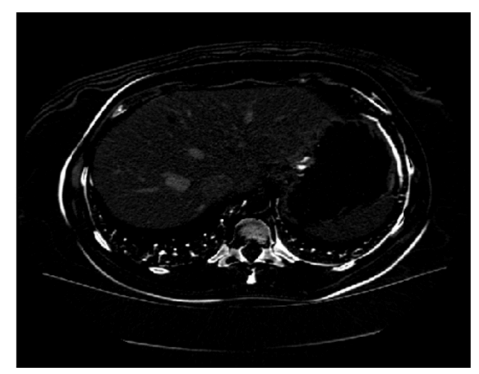

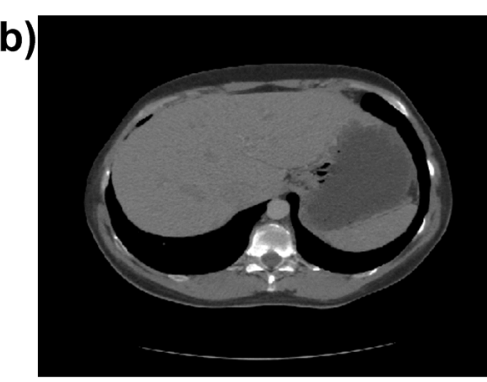

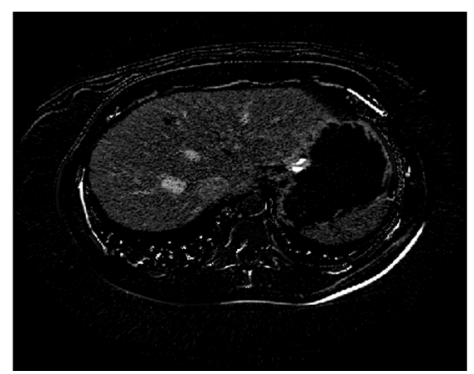

d)
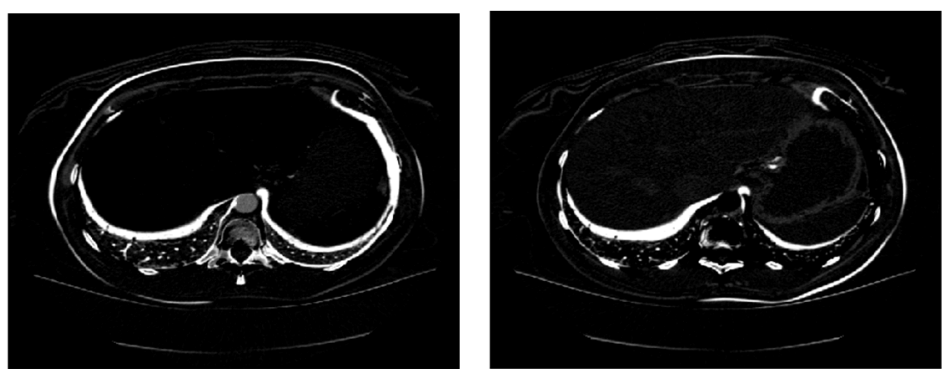

Figure 6. $\ell_{2}$-norm based NMU segmentation of the NBE three-phase CT image shown in Figure 5. Clinically and anatomically meaningful components were obtained and organized in four clusters where: a) vessels and rib are included; b) liver, spleen, spine and rib are included; c) spine and rib are included; d) lung is included.

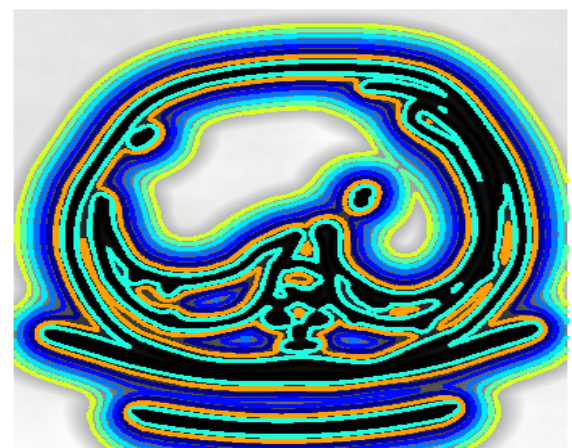

Figure 7. Isolines of the liver related segmented image of the second patient obtained by the NBE-NMU method (left in the cluster b) in Figure 6).

\section{CONCLUSION}

Methodology for contrast-enhanced unsupervised segmentation of the multi-phase CT image is proposed. The methodology relies on representation of the multi-phase CT image by a linear mixture model comprised of a product of a matrix of concentration profiles and matrix of spatial distributions of the organs present in the image. Hence, CT image segmentation is reduced to matrix factorization problem. Unsupervised segmentation method proposed in the paper combines nonlinear band expansion transform (that enhances contrast between organs by increasing dissimilarity between corresponding vectors of concentration profiles) and nonnegative matrix underapproximation that generates mutually sparse (non-overlapping) spatial distributions of the organs present in the image. Proposed method is demonstrated successfully on the two three-phase CT images of the liver. 


\section{ACKNOWLEDGEMENTS}

The work of I. Kopriva has been supported through grant 098-0982903-2558 funded by the Ministry of Science, Education and Sports, Republic of Croatia.

\section{REFERENCES}

[1] Hu, X., Shimizu, A., Kobatake, H., and Nawano, S., "Independent Component Analysis of Four-Phase Abdominal CT Images," Lecture Notes in Computer Science 3217, 916-924 (2004).

[2] Tao, G., Singh, A., and Bidaut, L., "Liver Segmentation from Registered Multiphase CT Data Sets with EM Clustering and GVF Level Set," Proc. of the SPIE Vol. 7623, 76230V:1-9 (2010).

[3] Kopriva, I., and Peršin, A., "Unsupervised decomposition of low-intensity low-dimensional multispectral fluorescent images for tumour demarcation", Medical Image Analysis 13, 507-518 (2009).

[4] Kopriva, I. and Cichocki, A., "Blind Multi-spectral Image Decomposition by 3D Nonnegative Tensor Factorization", Optics Letters 34, 2210-2212 (2009).

[5] Kopriva, I., and Cichocki, A., "Blind decomposition of low-dimensional multi-spectral image by sparse component analysis," Journal of Chemometrics 23, 590-597 (2009).

[6] Kopriva, I., Peršin, A., Puizina-Ivić, N., and Mirić, L. "Robust demarcation of basal cell carcinoma by dependent component analysis-based segmentation of multi-spectral fluorescence image," Journal Photochemistry and Photobiology B: Biology, 100, 10-18 (2010).

[7] Kopriva, I., and Jerić, I. "Blind separation of analytes in nuclear magnetic resonance spectroscopy and mass spectrometry: sparseness-based robust multicomponent analysis," Analytical Chemistry 82, 19111920 (2010).

[8] Kopriva, I., Jerić, I., and Smrečki, V. "Extraction of multiple pure component ${ }^{1} \mathrm{H}$ and ${ }^{13} \mathrm{C}$ NMR spectra from two mixtures: novel solution obtained by sparse component analysis-based blind decomposition," Analytica Chimica Acta 653, 143-153 (2009).

[9] Kopriva, I., and Jerić, I. "Multi-component Analysis: Blind Extraction of Pure Components Mass Spectra using Sparse Component Analysis," Journal of Mass Spectrometry 44, 1378-1388 (2009).

${ }^{[10]}$ Kopriva, I., Jerić, I. and Cichocki, A. "Blind Decomposition of Infrared Spectra Using Flexible Component Analysis," Chemometrics and Intelligent Laboratory Systems 97, 170-178 (2009).

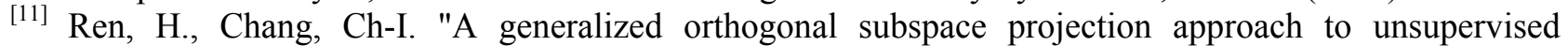
multispectral image classification," IEEE Trans. on Geosc. and Remote Sensing 38, 2515-2528 (2000).

${ }^{[12]}$ Ouyang, Y. C., Chen, H. M., Chai, J. W, Chen, C.C.C., Poon, S.K., Yang, C.W., Lee, S.K., and Chang, C.I.. "Band Expansion-Based Over-Complete Independent Component Analysis for Multispectral Processing of Magnetic Resonance Image," IEEE Trans. Biomed. Eng. 55, 1666-1677 (2008).

${ }^{[13]}$ Hyvärinen, A., "Independent component analysis for time-dependent stochastic processes," Proc. of the International Conference on Artificial Neural Networks (ICANN'98), 541-546 (1998).

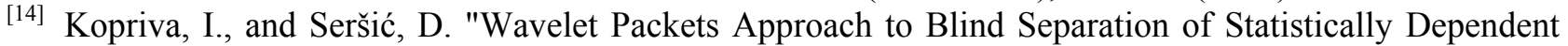
Sources," Neurocomputing 71, 1642-1655 (2008).

[15] Koldovský, Z., Tichavský, P., and Oja, E. "Efficient Variant of Algorithm for FastICA for Independent Component Analysis Attaining the Cramér-Rao Lower Bound," IEEE Trans. Neural Net. 17, 1265-1277 (2006).

[16] Gillis, N., and Glineur, F. "Using underapproximations for sparse nonnegative matrix factorization. Pattern Recognition 43, 1676-1687 (2010).

[17] Cichocki, A., Zdunek, R., Phan, A. H., and Amari, S. I., [Nonnegative Matrix and Tensor Factorization], John Wiley \& Sons (2009).

${ }^{[18]} \mathrm{Li}$, B., Acton, S. T. "Active contour external force using vector field convolution for image segmentation," IEEE Trans. Image Proc. 16, 2096-2106 (2007).

${ }^{[19]} \mathrm{Li}, \mathrm{B}$., and Acton, S. T. "Automatic Active Model Intialization via Poisson Inverse Gradient," IEEE Trans. Image Proc. 17, 1406-1420 (2008). 